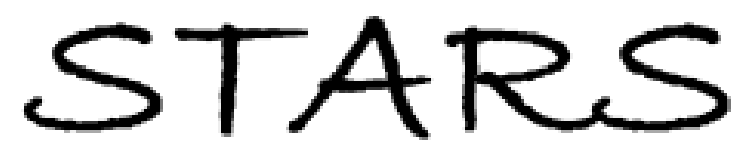

University of Central Florida

STARS

$1-1-2004$

\title{
Thermodynamic model of electric-field-induced pattern formation in binary dielectric fluids
}

\author{
M. D. Johnson \\ University of Central Florida \\ X. Duan \\ University of Central Florida \\ Brett Riley \\ University of Central Florida \\ Aniket Bhattacharya \\ University of Central Florida \\ Weili Luo \\ University of Central Florida \\ Find similar works at: https://stars.library.ucf.edu/facultybib2000 \\ University of Central Florida Libraries http://library.ucf.edu
}

This Article is brought to you for free and open access by the Faculty Bibliography at STARS. It has been accepted for inclusion in Faculty Bibliography 2000s by an authorized administrator of STARS. For more information, please contactSTARS@ucf.edu.

\section{Recommended Citation}

Johnson, M. D.; Duan, X.; Riley, Brett; Bhattacharya, Aniket; and Luo, Weili, "Thermodynamic model of electric-field-induced pattern formation in binary dielectric fluids" (2004). Faculty Bibliography 2000 s. 4465.

https://stars.library.ucf.edu/facultybib2000/4465

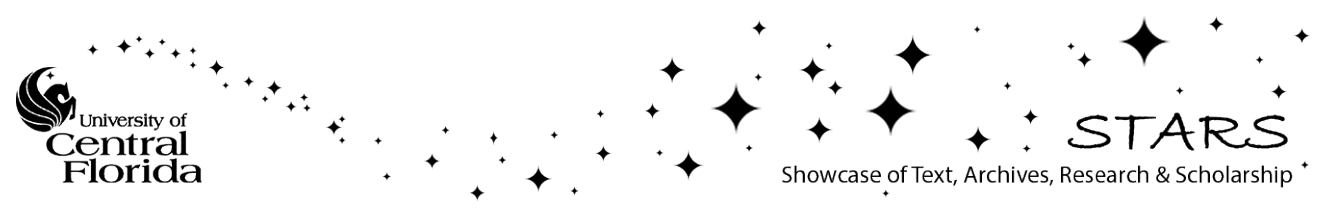




\title{
Thermodynamic model of electric-field-induced pattern formation in binary dielectric fluids
}

\author{
M. D. Johnson, ${ }^{*}$ X. Duan, Brett Riley, Aniket Bhattacharya, ${ }^{\dagger}$ and Weili Luo \\ Department of Physics, University of Central Florida, Orlando, Florida 32816-2385, USA
}

(Received 21 November 2002; revised manuscript received 7 January 2004; published 9 April 2004)

\begin{abstract}
An electric-field-induced phase transition and pattern formation in a binary dielectric fluid layer are studied using a coarse-grained free-energy functional. The electrostatic part of the free energy is a nonlinear functional of the dielectric function, which depends in turn on the local colloidal concentration. We determine the phase coexistence curve and find that beyond a critical electric field the system phase separates. Accompanying the phase separation are patterns similar to those observed in a spinodal decomposition of an ordinary binary fluid. The temporal evolution of the phase separating patterns are discussed both analytically and numerically by integrating a Cahn-Hilliard type of equation.
\end{abstract}

DOI: 10.1103/PhysRevE.69.041501

PACS number(s): 83.80.Gv, 83.80.Hj, 82.70.Dd, 89.75.Kd

\section{INTRODUCTION}

Systems undergoing a phase separation from a disordered phase to a more ordered state often form interesting patterns [1] as they select and approach the final state. The process of phase ordering, or coarsening dynamics, can be classified into different universality classes depending on the physical dimension, the dimension of the order parameter, and conservation laws $[2,3]$. Typically the associated scaling properties and dynamical universalities can be understood using a coarse-grained free-energy functional. A phenomenological equation of motion for the order parameter field using a time dependent Ginzburg-Landau approach then allows study of the evolving patterns [4].

Ferrofluids [5], which are colloidal suspensions of magnetic particles in solution, exhibit complex labyrinthine pattern formation when trapped between closely spaced glass plates and subjected to a transverse magnetic field [5-7]. The complex patterns result from competition between the surface tension and long-range forces, and have much in common with patterns observed in amphiphilic systems and Langmuir-Blodget films. In this paper we report on a similar effect in colloidal suspensions in which phase separation and a concomitant pattern formation are driven by an external electric field. Our results are based upon a macroscopic thermodynamic model of a dielectric binary liquid. This work was motivated by recent experiments demonstrating such behavior $[8,9]$, and shares some features in common with the experiments. We reported some preliminary results in Refs. $[9,10]$.

We consider colloids consisting of nanometer scale (typical diameter $\sim 10 \mathrm{~nm}$ ) dielectric particles suspended in a dielectric solution. The patterns examined here have length scales much larger than the particle size and the typical interparticle spacing. Consequently we use a coarse-grained free-energy functional and obtain the corresponding CahnHilliard equation [11-13] to study the evolving patterns in a colloidal suspension driven by an electric field. This is simi-

\footnotetext{
*Electronic address: mjohnson@ucf.edu

${ }^{\dagger}$ Electronic address: aniket@physics.ucf.edu

¥Electronic address: luo@ucf.edu
}

lar to the model B for a conserved scalar order parameter used to study phase separation in binary liquids [2-4]. In the present work the order parameter is the local particle concentration or volume fraction $c(\mathbf{r})(0 \leqslant c \leqslant 1)$. We model the particle interaction using a local dielectric constant $\varepsilon(c(\mathbf{r}))$ which depends nonlinearly on the local concentration. The use of a phenomenological dielectric constant $\varepsilon(c)$ with a positive curvature is an important ingredient of the model. It turns out that beyond a critical value of the electric field the electrostatic energy dominates and drives phase separation and pattern formation.

The approach developed in this paper is thermodynamic, based on a macroscopic free energy. Analyzing equilibrium properties yields a phase diagram which exhibits instabilities that can lead to pattern formation. Some understanding of the dynamics of pattern formation then comes from investigating the corresponding time-dependent Ginzburg-Landau equation. We begin by describing the model free energy and its equilibrium properties, and then turn to dynamics.

\section{MODEL FREE ENERGY}

Consider a binary dielectric liquid as described above placed between parallel capacitor plates separated by $d$. The liquid has capacitance

$$
C=\frac{1}{4 \pi d^{2}} \int d^{3} r \varepsilon(c(\mathbf{r}))
$$

When the internal energy is dominated by the electrostatic contribution, the Helmholtz free energy is

$$
F=\frac{1}{2} Q V-T S,
$$

where $V$ is the voltage between the plates, $T$ denotes the temperature, and $S$ represents the entropy. The equilibrium state is obtained by minimizing $F$ at constant $Q, T$. For our purposes it is more convenient to make a Legendre transformation to a different free energy:

$$
\Omega=F-Q V=-\frac{1}{2} Q V-T S .
$$


Equilibrium is found by minimizing $\Omega$ at constant $V, T$ (note that $V$ is voltage and not volume). We will refer to $\Omega$ as "the" free energy.

For the dielectric constant $\varepsilon$ we use a phenomenological model due to Lichteneker $[9,14]$ :

$$
\varepsilon(c)=\varepsilon_{f} e^{\gamma c}, \quad \text { where } \gamma=\ln \varepsilon_{p} / \varepsilon_{f} .
$$

This interpolates between the dielectric constant of pure solvent $\left(\varepsilon_{f}\right)$ and that of pure particle $\left(\varepsilon_{p}\right)$ as the concentration varies from $c=0$ to $c=1$. In this paper we use a physically reasonable value $\gamma=3[8,9]$. The specific choice of $\varepsilon(c)$ in Eq. (4) is not essential. The results we report turn out to be largely independent of the form of $\varepsilon(c)$, as long as it has positive curvature (a point we discuss later).

We use an approximate entropy functional appropriate for interacting particles at low concentration $[8,9,15,16]$ :

$$
S=-\frac{k_{B}}{v} \int d^{3} r\{c(\mathbf{r}) \ln c(\mathbf{r})+[1-c(\mathbf{r})] \ln [1-c(\mathbf{r})]\} .
$$

This expression can be obtained by a simple counting argument. In the absence of interactions $v$ is the particle volume and Eq. (5) is the entropy of free particles [17]. Here however $v$ is instead the so-called "correlation volume," which accounts phenomenologically for the particle interactions.

In a typical experiment the plate separation $d$ is so small that the concentration $c(\mathbf{r})$ can be treated as effectively two dimensional. Then we can let the coordinate $\mathbf{r}$ denote lateral position within the plane, with $\mathbf{r}=(x, y)$. In this limit the electric field $E$ becomes uniform $(E=V / d)$, and the free energy becomes [9]

$$
\Omega=d \int d^{2} r f(c(\mathbf{r}))
$$

where

$$
f(c)=-\frac{E^{2}}{8 \pi} \varepsilon(c)+\frac{k_{B} T}{v}[c \ln c+(1-c) \ln (1-c)] .
$$

For $\varepsilon(c)$ given in Eq. (4), the temperature and electric field naturally scale to the dimensionless form

$$
\widetilde{T}=\frac{k_{B} T / v}{\varepsilon_{f} E^{2} / 8 \pi}
$$

\section{EQUILIBRIUM PHASE SEPARATION}

We are interested in potential phase separations in the system. These can occur if $f(c)$ possesses inflection points [18]. Suppose that in equilibrium the system phase separates into volume fractions $x_{1,2}$ with concentrations $c_{1,2}$. If the average concentration is $c$, then these must satisfy

$$
\sum_{i=1}^{2} x_{i}=1, \quad \sum_{i=1}^{2} x_{i} c_{i}=c
$$

and so

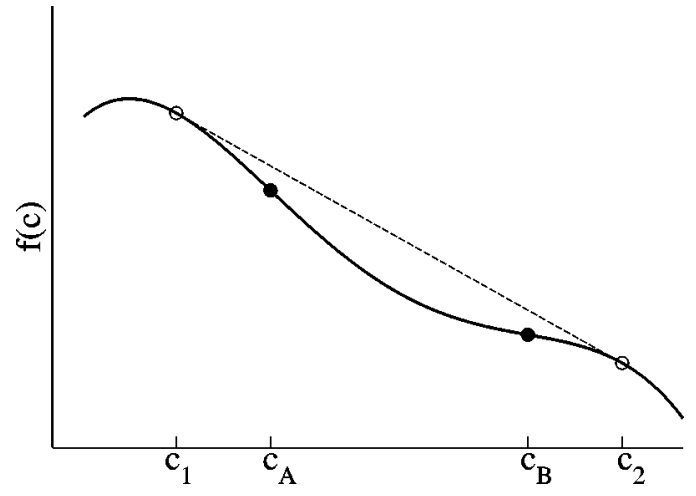

FIG. 1. Two points on a curve can have a common tangent only if the curve has at least two inflection points. The inflection points at $c_{A}, c_{B}$ are indicated by filled circles and the phase separation points at $c_{1}, c_{2}$ by open circles.

$$
x_{1}=\frac{c_{2}-c}{c_{2}-c_{1}}, \quad x_{2}=\frac{c-c_{1}}{c_{2}-c_{1}} \text {. }
$$

To find the conditions under which the equilibrium state is phase separated in this manner, we minimize the free energy $\sum_{i} x_{i} f\left(c_{i}\right)$ with respect to $c_{1}, c_{2}, x_{1}, x_{2}$, subject to the constraints, Eq. (8). Using Lagrange multipliers, this means minimizing

$$
\tilde{\Omega}=\sum_{i=1}^{2} x_{i}\left[f\left(c_{i}\right)-\mu c_{i}-\lambda\right]
$$

Except at the endpoints ( $x_{i}$ or $c_{i}$ equal to 0 or 1$)$ this minimization yields

$$
\begin{gathered}
f\left(c_{i}\right)=\mu c_{i}+\lambda, \\
f^{\prime}\left(c_{i}\right)=\mu .
\end{gathered}
$$

If $c_{1}$ and $c_{2}$ both satisfy Eq. (11a), then

$$
f\left(c_{2}\right)-f\left(c_{1}\right)=\mu\left(c_{2}-c_{1}\right) .
$$

This result is summarized by a simple geometrical construction illustrated in Fig. 1. If the straight line joining the points $\left[c_{1}, f\left(c_{1}\right)\right]$ and $\left[c_{2}, f\left(c_{2}\right)\right]$ is tangent to $f(c)$ at both points, then phase separation will occur for $c_{1} \leqslant c \leqslant c_{2}$. This can occur only if $f(c)$ has two or more inflection points (see Fig. 1). For our model, the endpoints $c=0,1$ never correspond to a minimum free-energy state.

Consider now the free-energy density $f(c)$ for our dielectric fluid, Eq. (6b). The entropic term $-T S$ has positive curvature. At high temperatures this term dominates, $f(c)$ has positive curvature everywhere, and the equilibrium state is homogeneous. This is illustrated by the highest-temperature trace $(\widetilde{T}=20)$ in Fig. 2, which uses the model dielectric function Eq. (4). As the temperature is lowered the electrostatic energy term $-Q V / 2=-E^{2} \varepsilon(c) / 8 \pi$ plays an increasingly important role. When $\varepsilon(c)$ has positive curvature, the electrostatic term has negative curvature, and at low tem- 

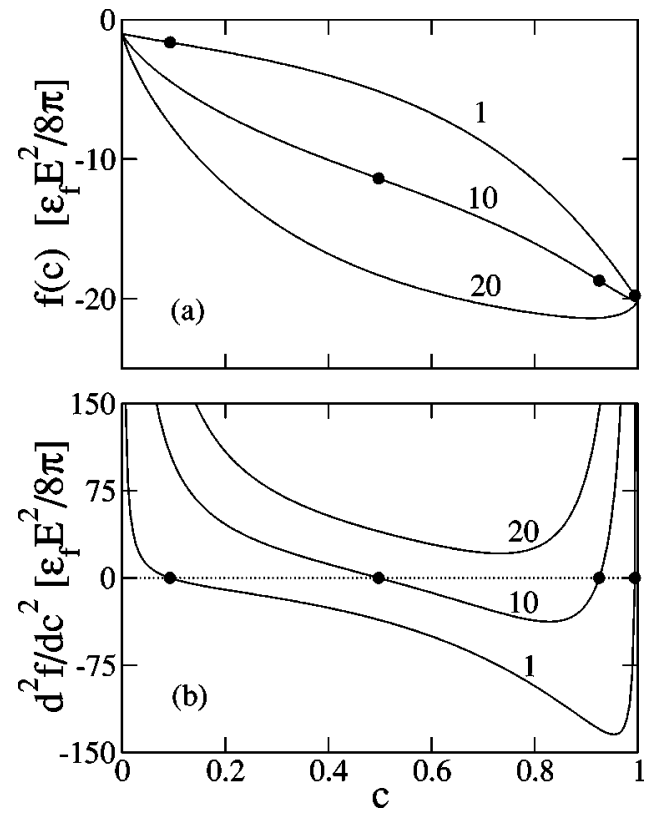

FIG. 2. (a) $f(c)$ vs $c$ for the three scaled temperatures $\widetilde{T}$ $=1,10,20$, using Eqs. (4) and (6b) with $\gamma=3$. At the two lower temperatures two inflection points are present. These are more easily seen in (b) $f^{\prime \prime}(c)$ vs $c$. The inflection points are indicated by filled circles.

peratures this term leads to inflection points and phase separation. This is illustrated by the two lower-temperature traces in Fig. 2.

The phase diagram for the free energy, Eq. (6b), developed using the above procedure, is shown in Fig. 3 for $\gamma=3$. Below the coexistence curve (the solid line) the homogeneous phase becomes metastable and in equilibrium the system phase separates. This curve is the locus of points $c_{1,2}$ obtained from solving Eq. (11) at all temperatures. Although there is no exact expression for the coexistence temperature, for the physically important case of low concentrations we find approximately

$$
\widetilde{T}_{\mathrm{coex}} \approx \frac{(1+\gamma-\gamma c) e^{\gamma c}-e^{\gamma}}{\ln c} .
$$

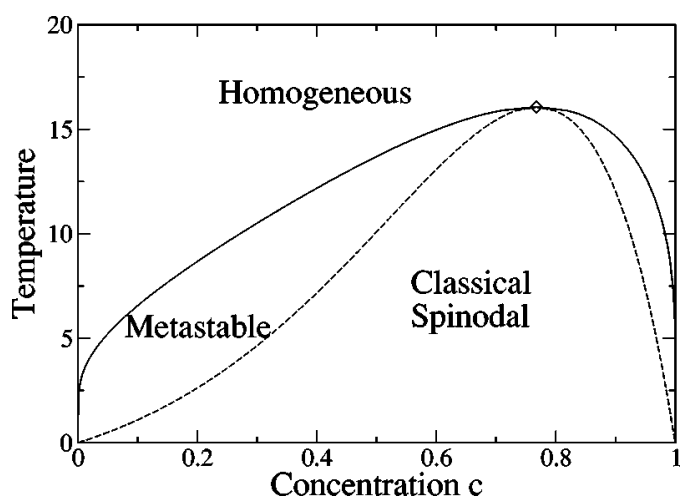

FIG. 3. Phase diagram for the free energy given in Eqs. (4) and (6b) for $\gamma=3$. Temperature is $\widetilde{T}$. The coexistence curve $\widetilde{T}_{\text {coex }}$ (solid) and the spinodal $\widetilde{T}_{s}$ (dashed) are shown. The critical point, Eq. (15), is marked by a diamond.
For $\gamma=3$ this is accurate for concentrations $c \leqq 0.3$.

Lying below the dashed phase boundary in Fig. 3 is the classical spinodal region, where the homogeneous state becomes unstable $\left[f^{\prime \prime}(c)<0\right]$. This phase boundary is given by the condition $f^{\prime \prime}(c)=0$, which yields

$$
\widetilde{T}_{s}=\gamma^{2} e^{\gamma c} c(1-c) .
$$

The coexistence and spinodal curves touch at a critical point (marked by a diamond in Fig. 3). This occurs at the critical concentration $c_{c}$ and critical temperature $T_{c}$ where the two inflection points present at lower temperatures coincide [and so $f^{\prime \prime}\left(c_{c}\right)=0=f^{\prime \prime \prime}\left(c_{c}\right)$ ]. For the model dielectric function, Eq. (4), the critical values are

$$
\begin{gathered}
c_{c}=\frac{1}{2 \gamma}\left(\gamma-2+\sqrt{\gamma^{2}+4}\right), \\
\widetilde{T}_{c}=\gamma^{2} e^{\gamma c^{c} c_{c}}\left(1-c_{c}\right) .
\end{gathered}
$$

The instabilities give the possibility of pattern formation, which we now discuss.

\section{PATTERN FORMATION DYNAMICS}

Pattern formation after quenches into the metastable or spinodal regions provides important insight into the underlying mechanisms for phase transformation in the system. For a system like the one modeled here, it is experimentally easier to increase the voltage suddenly while holding the temperature fixed; by Eq. (7) this is equivalent to a quench. Here we investigate pattern formation under quenches using a Cahn-Hilliard-Cook type of equation developed from the free energy [11-13].

Notice first that when inhomogeneities arise the free energy needs an additional $|\nabla c|^{2}$ term [11]. This can be viewed as the surface energy between domains of different concentration; such terms arise generally in any interacting system. Consequently in this section we write the free energy

$$
\Omega=\int d^{3} r\left[f(c(\mathbf{r}))+\frac{1}{2} \kappa|\nabla c|^{2}\right],
$$

where $\kappa$ is a phenomenological parameter.

The concentration current density is obtained from the local chemical potential $\mu$,

$$
\mathbf{j}(\mathbf{r})=-M v_{0} \nabla \mu(\mathbf{r}),
$$

where $M$ is the mobility (taken to be constant) and $v_{0}$ is the volume of the colloidal particle. Combining this with the continuity equation $\dot{c}+\boldsymbol{\nabla} \cdot \mathbf{j}=0$ yields the well-known CahnHilliard equation $[11-13]$

$$
\frac{\partial c}{\partial t}=M v_{0} \nabla^{2} \mu
$$

The local chemical potential is obtained from the free energy: 


$$
\mu(\mathbf{r})=\left.v_{0} \frac{\delta \Omega}{\delta c(\mathbf{r})}\right|_{T E}=v_{0}\left[f^{\prime}(c(\mathbf{r}))-\kappa \nabla^{2} c(\mathbf{r})\right] .
$$

Inserting this into Eq. (18) gives

$$
\frac{\partial c}{\partial t}=M v_{0}^{2}\left[\nabla^{2} f^{\prime}(c(\mathbf{r}))-\kappa \nabla^{4} c(\mathbf{r})\right] .
$$

The free-energy density $f(c)$ is in Eqs. (4) and (6b). A convenient dimensionless form comes from writing time in units of $\tau$ and length in units of $\lambda$, where

$$
\begin{gathered}
\tau=\frac{\kappa}{M v_{0}^{2}}\left(\frac{8 \pi}{\varepsilon_{f} E^{2}}\right)^{2}, \\
\lambda=\sqrt{\frac{8 \pi \kappa}{\varepsilon_{f} E^{2}}} .
\end{gathered}
$$

With these rescalings Eq. (20) becomes

$$
\frac{\partial c}{\partial t}=\nabla^{2}\left[\tilde{f}^{\prime}(c)-\nabla^{2} c\right],
$$

where

$$
\widetilde{f}(c)=-e^{\gamma c}+\widetilde{T}[c \ln c+(1-c) \ln (1-c)] .
$$

The Cahn-Hilliard equation (22a) allows the study of pattern formation. In this particular case the free energy, Eq. (22b), was constructed to be directly relevant to experiments on dielectric colloidal fluids $[8,9]$. We study pattern formation after quenches by solving Eq. (22a) starting from a uniform distribution (plus a small random component) [19].

One can get some intuition about dynamics by linearizing the Cahn-Hilliard equation [2]. The result is accurate for early times, before the nonlinear terms become significant. Write $c(\mathbf{r})=c+\delta c(\mathbf{r})$, where $c$ is the average concentration. For small $\delta c$ expand

$$
\widetilde{f}(c+\delta c)=\widetilde{f}(c)+\tilde{\mu}_{0} \delta c-\frac{1}{2} b(\delta c)^{2}+\cdots,
$$

where

$$
\begin{aligned}
\widetilde{\mu}_{0}=\widetilde{f}^{\prime}(c) & =-\gamma e^{\gamma c}+\widetilde{T}[\ln c-\ln (1-c)], \\
b & =-\widetilde{f}^{\prime \prime}(c)=\frac{\widetilde{T}_{s}-\widetilde{T}}{c(1-c)} .
\end{aligned}
$$

$\widetilde{T}_{s}$ is given in Eq. (14). The linear and constant terms in Eq. (23) play no role in the dynamics. Keeping only the secondorder term from Eq. (23), the free energy, Eq. (16), becomes

$$
\Omega \approx \frac{1}{2} \int d^{3} r\left[\kappa|\nabla \delta c|^{2}-b(\delta c)^{2}\right] .
$$

This expansion connects our model to the coarsening dynamics of model B, an idealized description of a binary alloy with a conserved scalar order parameter $\phi(\mathbf{r})$. The free energy for such a system is generally assumed to have the Ginzburg-Landau form

$$
F=\frac{1}{2} \int d^{3} r\left[\kappa|\nabla \phi|^{2}-b \phi^{2}+\frac{u}{2} \phi^{4}\right],
$$

where $\kappa$ and $u$ are assumed to be positive. Below the critical temperature $b$ becomes positive, yielding a broken symmetry. Higher-order terms beyond $\phi^{4}$ are irrelevant for the dynamic universality class. In fact, the early-time dynamics are dominated by $\phi^{2}$ terms. From the correspondence between Eqs. (25) and (26) we expect our model to have early-time dynamics very similar to those of model B.

Using the expansion, Eq. (25) linearizes the diffusion equation Eq. (22):

$$
\frac{\partial(\delta c)}{\partial t}=-\nabla^{2}\left[b \delta c+\nabla^{2} \delta c\right] .
$$

This is solved by expanding the fluctuation $\delta c$ in Fourier components:

$$
\delta c(\mathbf{r}, t)=\int \frac{d^{2} k}{(2 \pi)^{2}} e^{i \mathbf{k} \cdot \mathbf{r}} c(\mathbf{k}, t) .
$$

We find

$$
c(\mathbf{k}, t)=c(\mathbf{k}, 0) e^{\alpha_{k} t}, \quad \text { where } \alpha_{k}=k^{2}\left(b-k^{2}\right) .
$$

Outside of the spinodal region $\widetilde{T}>\widetilde{T}_{s}$ and so by Eq. (24b) $b<0$. Consequently all components $c(\mathbf{k}, t)$ decay. This describes the homogeneous equilibrium state: fluctuations away from a uniform concentration decay.

However, within the classical spinodal region $b>0$. Thus long wavelength modes, those with wave vector $k<\sqrt{b}$, grow exponentially. The most rapid growth occurs for $k_{m}$ $=\sqrt{b / 2}$, where $\alpha_{k}$ reaches its maximum value $b^{2} / 4$. Thus in the classical spinodal regime, where the homogeneous state is unstable, early-time exponential growth leads to structures a typical (dimensionless) length scale $k_{m}^{-1} \sim\left(\widetilde{T}_{s}-T\right)^{-1 / 2}$. These structures grow exponentially in time with a (dimensionless) time scale $1 / \alpha_{k_{m}}=4 / b^{2} \sim\left(\widetilde{T}_{s}-\widetilde{T}\right)^{-2}$.

For example, consider the structure factor

$$
S(\mathbf{k}, t)=\langle c(\mathbf{k}, t) c(-\mathbf{k}, t)\rangle .
$$

Here angle brackets represent a statistical average. Suppose that the initial fluctuations $c(\mathbf{k}, 0)$ are small and uncorrelated:

$$
\left\langle c(\mathbf{k}, 0) c\left(\mathbf{k}^{\prime}, 0\right)\right\rangle=A^{2} \delta^{2}\left(\mathbf{q}-\mathbf{q}^{\prime}\right) .
$$

Then for early times

$$
S(\mathbf{k}, t)=A^{2} e^{2 \alpha_{k} t} .
$$

This grows ever more peaked at $k_{m}$ as time increases. Fourier transforming gives the real-space correlation function 


$$
\left\langle\delta c(\mathbf{r}, t) \delta c\left(\mathbf{r}^{\prime}, t\right)\right\rangle \sim \frac{A^{2}}{t^{1 / 2}} e^{b^{2} t / 4} J_{0}\left(k_{m}\left|\mathbf{r}-\mathbf{r}^{\prime}\right|\right)
$$

which exhibits the length and time scales described above.

Including dimensions, the structures developing in early times have a length scale

$$
L \sim \frac{\lambda}{k_{m}}=\sqrt{\frac{8 \pi \kappa}{\varepsilon_{f} E^{2}}} \sqrt{\frac{2 c(1-c)}{\widetilde{T}_{s}-\widetilde{T}}}
$$

and grow with a time scale

$$
t_{0} \sim \frac{4 \tau}{b^{2}}=\frac{\kappa}{M v_{0}^{2}}\left(\frac{8 \pi}{\varepsilon_{f} E^{2}}\right)^{2}\left[\frac{2 c(1-c)}{\widetilde{T}_{s}-\widetilde{T}}\right]^{2}
$$

Deep quenches into the classical spinodal region should lead to dynamically developing structures on a length scale $L$ given by Eq. (34). These structures result from a longwavelength instability. They represent not the equilibrium state, but the early-time evolution from the highly nonequilibrium homogeneous state toward the (eventual) phaseseparated equilibrium. The linearized description is accurate for very small initial times. Soon the fluctuations grow large enough for the nonlinear terms to play a role, and we turn to numerical means for the solution of Eq. (22).

\section{NUMERICAL INTEGRATION OF THE DIFFUSION EQUATION}

We numerically integrate the lattice discretized version of Eqs. (22) using a first-order Euler scheme, choosing step sizes to avoid unphysical instabilities [20]. This algorithm is adequate for times during which the system begins to organize into patterns. To carry the simulation all the way to phase separation requires a more sophisticated approach [21]. However the model we use also omits hydrodynamic effects which may be important for the late time coarsening process. Consequently here we present results during the era of pattern formation only.

The initial configuration consists of a uniform concentration $c$ plus a small random component. Starting from this initial state, Eq. (22) is integrated for various values of $\widetilde{T}$. In agreement with the results of the previous sections we find that phase separation occurs below the coexistence temperature $\widetilde{T}_{\text {coex }}$ (or, equivalently, above a threshold electric field). In the phase-separation region the concentration develops labyrinthine patterns consisting of stripes of higher and lower concentration.

Typical results for a quench deep into the phase separation region are shown in Figs. 4 and 5. The latter clearly shows that a labyrinthine pattern has developed.

We monitor two quantities during the simulation. At each time step we search for the maximum and minimum concentrations, and define a contrast parameter $\left(c_{\max }-c_{\min }\right) / 2 c$. This parameter indicates the visibility of the developing pattern. Another quantity of interest is the pair correlation function

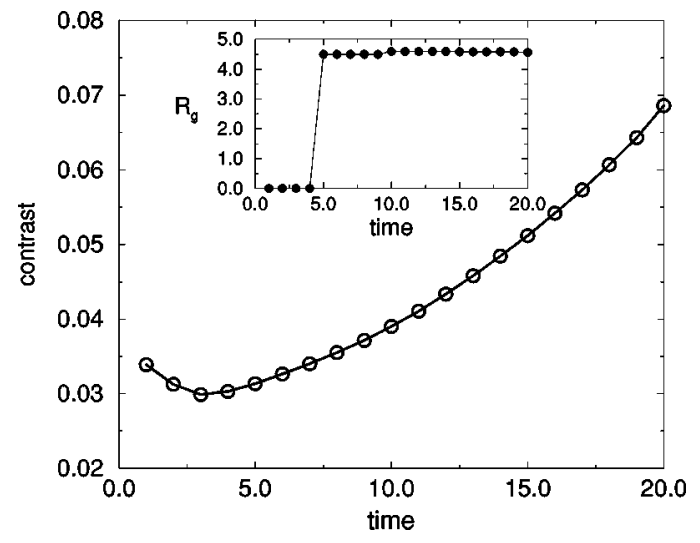

FIG. 4. Early stage increase in contrast $\left(c_{\max }-c_{\min }\right) / 2 c$ and domain size (inset) as a function of time. Here time and space are in the units of Eq. (21). The simulation was done on a $128 \times 128$ lattice with an initial random configuration \pm 0.01 around $c=0.1$ for $\gamma=1.0$ and $\widetilde{T}=0.045$.

$$
G(\mathbf{r}, t)=\left\langle\frac{1}{V} \int[c(\mathbf{r}, t)-c]\left[c\left(\mathbf{r}+\mathbf{r}^{\prime}, t\right)-c\right] d^{2} \mathbf{r}^{\prime}\right\rangle
$$

The average domain width $R_{g}$ can be calculated from the first zero of the normalized pair correlation function $\widetilde{G}(\mathbf{r}, t)$ $=G(\mathbf{r}, t) / G(0, t)$. The upper panel of Fig. 4 shows the earlytime variation of the contrast parameter and the domain width $R_{g}$ as a function of the scaled time. The average domain width after a rapid initial increase saturates at this temperature to a value $\sim 4.5 \lambda$.

\section{SUMMARY}

Finally we summarize the results of this paper, emphasizing experimental comparisons and predictions. The model, thermodynamic in nature, describes a dielectric colloidal suspension using a coarse-grained free-energy functional. The free energy contains two essential ingredients: an electrostatic energy and an entropy term, both expressed as nonlinear functionals of the spatially varying colloidal concentra-

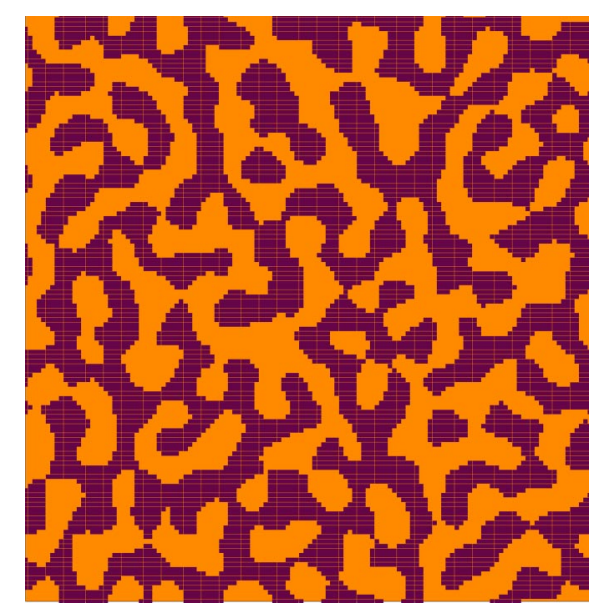

FIG. 5. A typical snapshot at time 20. Concentrations greater (less) than the average $c$ are dark (light). 
tion. This model shares features of a Ginzburg-Landau free energy that describes the phase separation of a binary mixture. Certain other features which would require a more complicated order parameter have been omitted, e.g., the orientational ordering arising from a magnetic dipolar interaction in the case of ferrocolloids.

Several features of this model are in good qualitative agreement with existing experiments on dielectric colloidal suspensions $[8,9]$. (1) The model clearly shows phase separation and pattern formation driven by an electric field. One distinctive feature seen in both experiment and this theory is the existence of a threshold critical field above which the instability starts. (2) The resulting patterns exhibit a typical labyrinthine morphology very similar to those observed experimentally. (3) In the numerically obtained patterns the stripes of high and low concentration have similar widths, in agreement with experiment.

This model also yields several predictions that can be tested by future experiments. Of note is the scaling of field and temperature in the form $E^{2} / T$ [Eq. (7)]. By dimensional analysis this will occur whenever the phase separation is driven by electrostatics. For the particular model used here, the threshold coexistence field above which phase separation occurs is [Eqs. (7) and (13)]

$$
\begin{aligned}
E_{\mathrm{coex}} & =\sqrt{\frac{8 \pi}{\varepsilon_{f}} \frac{k_{B} T}{v} \frac{1}{\widetilde{T}_{\mathrm{coex}}}} \\
& \approx \sqrt{\frac{8 \pi}{\varepsilon_{f}} \frac{k_{B} T}{v} \frac{\ln c}{(1+\gamma-\gamma c) e^{\gamma c}-e^{\gamma}}} .
\end{aligned}
$$

The threshold field here is seen to vary with temperature as $T^{1 / 2}$. This will occur whenever the electrostatic energy drives the phase separation, because of scaling.

A deep quench into the spinodal region leads to pattern formation with a characteristically labyrinthine morphology. This is a general result independent of most details of the free energy used here. For our model in particular the spinodal threshold electric field is [Eqs. (7) and (14)]

$$
E_{s}=\sqrt{\frac{8 \pi}{\varepsilon_{f}} \frac{k_{B} T}{v} \frac{1}{\widetilde{T}_{s}}}=\sqrt{\frac{8 \pi}{\varepsilon_{f}} \frac{k_{B} T}{v} \frac{1}{\gamma^{2} e^{\gamma c} c(1-c)}} .
$$

This threshold field also exhibits the $T^{1 / 2}$ temperature dependence which occurs for electric-field-driven phase transitions.

A characteristic feature of the dynamics of our model is that quenches into the spinodal region lead to very fast pattern formation. This is evident in Fig. 4. Our analytic examination of the early-time dynamics in Sec. IV showed characteristic length and time scales for pattern formation in this regime [Eqs. (34) and (35)]. Explicitly showing the electricfield dependence, the early-time patterns have a length scale

$$
L \sim \sqrt{\frac{2 \kappa v E_{s}^{2} c(1-c)}{k_{B} T\left(E^{2}-E_{s}^{2}\right)}}
$$

and grow with a time scale

$$
t_{0} \sim \frac{\kappa}{M v_{0}^{2}}\left[\frac{2 v E_{s}^{2} c(1-c)}{k_{B} T\left(E^{2}-E_{s}^{2}\right)}\right]^{2} .
$$

The electric-field dependence of the length scale was not observed in experiment. However, notice that both scales diverge at threshold. Rapid early-time growth with these characteristic length and time scales might be difficult to observe experimentally, since the patterns in this early-time regime have very small contrast. On the other hand, a leak current has been found in the experiment, and whether this has something to do with the field dependence of the length scale in these patterns is under experimental investigation.

The above discussion and the analytic and numerical studies of dynamics in this paper have emphasized the behavior under deep quenches, that is, quenches into the spinodal region. Shallower quenches into the metastable region (between the coexistence and spinodal lines in Fig. 3, i.e., for fields above $E_{\text {coex }}$ but below $E_{s}$ ) should also lead to pattern formation, but via nucleation rather than spinodal decomposition. Further studies in the metastable regime require substantial improvements in numerical algorithms. We expect to investigate this interesting regime in the future.

The particular dependences of the two threshold fields $E_{s}, E_{\text {coex }}$ on average concentration $c$ and the material parameter $\gamma$ are somewhat specific to our model. At low concentrations $E_{s}$ in Eq. (38) varies as $c^{-1 / 2}$. This is probably reliable, since it can be traced back to our entropy expression Eq. (5), which in turn is most accurate at low concentrations. At low concentrations $E_{\text {coex }}$ in Eq. (37) varies as $(\ln c)^{1 / 2}$. Experimentally it would be difficult to distinguish these two threshold fields since for fields above $E_{\text {coex }}$ but approaching $E_{s}$ pattern formation might look similar to spinodal decomposition.

Equation (38) also shows that at low concentrations $E_{s}$ $\sim 1 / \gamma=1 / \ln \left(\varepsilon_{p} / \varepsilon_{f}\right)$. This captures the intuitively reasonable feature that the threshold field decreases when the colloid and solvent have increasingly different dielectric constants. The coexistence threshold field $E_{\text {coex }}$ also decreases under these circumstances.

The phenomenology used here is most accurate at low particle concentrations. In particular, the entropy is essentially that of free particles, modified to take interactions into account via the phenomenological correlation volume $v$. Experiments with low overall concentrations should be described with reasonable accuracy by this model. The particular functional used for the electrostatic energy is of minor import. As long as the functional dependence of the dielectric constant on particle concentration has positive curvature essentially the same results are obtained.

\section{ACKNOWLEDGMENTS}

We thank Geoff Canright and John Evans for helpful conversations. We acknowledge support from the NSF through Grants Nos. DMR99-72683 (M.D.J.), DMR00-72901 (W.L.), and NSF-NIRT (ENG/ECS and CISE/EIA) Grant No. 0103587 (A.B. and W.L.). 
[1] For a review of pattern formation, see M.C. Cross and P.C. Hohenberg, Rev. Mod. Phys. 65, 851 (1993).

[2] J. D. Gunton, M. San Miguel, and P. S. Sahni, in Phase Transition and Critical Phenomena, edited by C. Domb and J. L. Lebowitz (Academic, London, 1983), Vol. 8.

[3] A.J. Bray, Adv. Phys. 43, 357 (1994).

[4] P.C. Hohenberg and B.I. Halperin, Rev. Mod. Phys. 49, 435 (1977).

[5] R. E. Rosenweig, Ferrohydrodynamics (Dover, New York, 1997).

[6] A.J. Dickinson, S. Erramilli, R. Goldstein, D.P. Jackson, and S.A. Langer, Science 261, 1012 (1993).

[7] H. Wang, Y. Zhu, C. Boyd, W. Luo, A. Cebers, and R.E. Rosensweig, Phys. Rev. Lett. 72, 1929 (1994).

[8] Xiaodong Duan and Weili Luo, Int. J. Mod. Phys. B 15, 837 (2001)

[9] Xiaodong Duan, Weili Luo, B. Wacase, and R.C. Davis, e-print cond-mat/0105484.

[10] Brett Riley, Aniket Bhattacharya, M. D. Johnson, X. Duan, and W. Luo, in Electrorheological Fluids and Magnetorheological Suspensions: Proceedings of the Eighth International Confer- ence, Nice, France, 2001, edited by G. Bossis (World Scientific, Singapore, 2002); Int. J. Mod. Phys. B 16, 2041 (2002).

[11] J.W. Cahn, J. Chem. Phys. 42, 93 (1965).

[12] J.W. Cahn and J.E. Hilliard, J. Chem. Phys. 28, 258 (1958).

[13] H.E. Cook, Acta Metall. 18, 297 (1970).

[14] C. Brosseau, J. Appl. Phys. 75, 672 (1994).

[15] R.P. Sear, Phys. Rev. E 56, 4463 (1997).

[16] J. Bibette, D. Roux, and F. Nallet, Phys. Rev. Lett. 65, 2470 (1990).

[17] C. Kittel and H. Kromer, Thermal Physics, 2nd ed. (Freeman, San Francisco, 1998), Chap. 11.

[18] M. Plischke and B. Bergersen, Equilibrium Statistical Mechanics, 2nd ed. (World Scientific, Singapore, 1994).

[19] With the inclusion of a noise term the Cahn-Hilliard equation is called the Cahn-Hilliard-Cook equation [13] or model B in the classification of Hogenberg and Halperin [4].

[20] A. Chakrabarti, R. Toral, and J.D. Gunton, Phys. Rev. B 39, 4386 (1989).

[21] A. Chakrabarti, R. Toral, and J.D. Gunton, Phys. Rev. E 47, 3025 (1993). 